\title{
An Algebraic Substructuring Method for High-Frequency Response Analysis of Micro-systems
}

\author{
Jin Hwan $\mathrm{Ko}^{1}$ and Zhaojun Bai ${ }^{2}$ \\ ${ }^{1}$ Dept. of Aerospace Information Engineering, Konkuk University, Seoul, Korea \\ jhko@invest.konkuk.ac.kr, jhko@cs.ucdavis.edu \\ ${ }^{2}$ Dept. of Computer Science, University of California, Davis CA 95616, USA \\ bai@cs.ucdavis.edu
}

\begin{abstract}
High-frequency response analysis (Hi-FRA) is required to predict the resonant behavior of modern microsystems operated over a high frequency range. Algebraic substructuring (AS) method is a powerful numerical technique for FRA. However, the existing AS method is developed for low-FRA, say over the range $1 \mathrm{~Hz}-2 \mathrm{KHz}$. In this work, we extend the AS method for FRA over a given frequency range $\left[\omega_{\min }, \omega_{\max }\right]$. Therefore, it can be efficiently applied to systems operated at high frequency, say over the range $1 \mathrm{MHz}-2 \mathrm{MHz}$. The success of the proposed method is demonstrated by Hi-FRA of a microgyroscope.
\end{abstract}

Keywords: High-Frequency Response Analysis, Algebraic Substructuring, Micro-Systems, Frequency Sweep Algorithm.

\section{Introduction}

Frequency Response Analysis (FRA) studies structural responses to steady-state oscillatory excitation to predict the resonant behavior in an operation (excitation) range of frequencies. Resonant sensors in microelectromechanical systems (MEMS) and other microscale structures are designed to catch the resonant behavior over a higher frequency range. Therefore, the Hi-FRA is typically required for the microscale structures.

The discretized model of a structure we consider in this paper is a continuous single-input single-output second-order system of the form

$$
\left\{\begin{aligned}
M \ddot{x}(t)+D \dot{x}(t)+K x(t) & =b u(t) \\
y(t) & =l^{T} x(t)
\end{aligned}\right.
$$

with the initial conditions $x(0)=x_{0}$ and $\dot{x}(0)=v_{0}$. Here $t$ is the time variable, $x(t) \in \mathcal{R}^{N}$ is a state vector, $N$ is the degree of freedom (DOF). $u(t)$ is the input excitation force and $y(t)$ is the output measurement function. $b \in \mathcal{R}^{N}$ and $l \in \mathcal{R}^{N}$ are the input and output distribution vectors. $M, K, D \in \mathcal{R}^{N \times N}$ are system mass, stiffness and damping matrices. It is assumed that the $M$ and $K$ 
are symmetric positive definite. The input-output behavior of the model (1) is characterized by the transfer function

$$
H(\omega)=l^{T}\left(-\omega^{2} M+i \omega D+K\right)^{-1} b,
$$

where $\omega$ is the frequency and $i=\sqrt{-1}$. Mathematically, the low FRA is on the computation of the transfer function $H(\omega)$ for $\omega$ over the range $\left[1, \omega_{\max }\right]$, where $\omega_{\max }$ is small, say at KHz. The Hi-FRA is about the computation of $H(\omega)$ for $\omega$ over the range $\left[\omega_{\min }, \omega_{\max }\right]$, where $\omega_{\min }$ and $\omega_{\max }$ are large, say at $\mathrm{MHz}$.

Due to the large DOF of the model (1), it is prohibitive to directly compute $H(\omega)$ over a large number of frequency points $\omega_{k}$ over the range of interest. A popular approach of the FRA is based on an eigensystem analysis, called the mode superposition (MS) method. One first extracts $n$ eigenpairs $\left(\lambda_{k}, q_{k}\right)$ of the matrix pair $(K, M)$ :

$$
K q_{k}=\lambda_{k} M q_{k},
$$

where $q_{k}^{T} K q_{k}=\lambda_{k}$ and $q_{k}^{T} M q_{k}=1$. Then by projecting the transfer function $H(\omega)$ onto the subspace $\operatorname{span}\left\{Q_{n}\right\}=\operatorname{span}\left\{\left[q_{1}, q_{2}, \ldots, q_{n}\right]\right\}$, it yields

$$
H_{n}(\omega)=l_{n}^{T}\left(-\omega^{2} I_{n}+\mathrm{i} \omega D_{n}+\Lambda_{n}\right)^{-1} b_{n},
$$

where $\Lambda_{n}=\operatorname{diag}\left(\lambda_{1}, \ldots, \lambda_{n}\right), D_{n}=Q_{n}^{T} D Q_{n}, l_{n}=Q_{n}^{T} l$ and $b_{n}=Q_{n}^{T} b$. The shift-and-invert Lanczos (SIL) method as an eigensolver has been the method of choice for decades. However, the continual and compelling need for the FRA of very large model (1) challenges the computational efficiency of the method. Substructuring approaches, initially developed in early 1960s, are being studied in recent years. The automated multi-level substructuring (AMLS) method [12] is one of substructuring approaches, in which the structure is recursively divided of many of subdomains, and these subdomains can be handled efficiently and in parallel. An algebraic analysis of the AMLS method, referred to as the algebraic structure (AS) method, is studied in 10,5. The AMLS has been successfully used for low FRA in which the smallest eigenmodes are required 2. However, the direct application of the AMLS to the Hi-FRA would require a large number of eigenmodes starting from the smallest to the large ones to match the high frequencies. It is computationally inefficient. In this paper, we propose an extension of the AMLS method for Hi-FRA application. Since the implementation of the AMLS is a proprietary software, we will use the AS method presented in [10.5] as an eigensolver, and then present the FRA method that is the an extension of the AMLS frequency sweeping algorithm [2].

\section{Algebraic Substructuring}

For Hi-FRA, the eigenmodes corresponding to the natural frequencies closest to the operation range are most important. Hence, we begin with a shifted eigenproblem of (3):

$$
K^{\sigma} q=\lambda^{\sigma} M q
$$


where $K^{\sigma}=K-\sigma M$ and $\lambda^{\sigma}=\lambda-\sigma . \sigma$ is a prescribed shift related to the frequency range $\left[\omega_{\min }, \omega_{\max }\right]$. The choice of the shift $\sigma$ is to be discussed later. We assume that the matrix pair $\left(K^{\sigma}, M\right)$ is of the partition

$$
K^{\sigma}=\begin{gathered}
N_{1} \\
N_{2} \\
N_{2} \\
N_{3}
\end{gathered}\left[\begin{array}{lll}
K_{11}^{\sigma} & & K_{13}^{\sigma} \\
& K_{22}^{\sigma} & K_{23}^{\sigma} \\
K_{31}^{\sigma} & K_{32}^{\sigma} & K_{33}^{\sigma}
\end{array}\right], \quad M=\begin{gathered}
N_{1} \\
N_{2}
\end{gathered}\left[\begin{array}{ccc}
M_{11} & & M_{13} \\
& M_{22} & M_{23} \\
M_{31} & M_{32} & M_{33}
\end{array}\right],
$$

where $\left(K_{11}^{\sigma}, M_{11}\right)$ and $\left(K_{22}^{\sigma}, M_{22}\right)$ are two substructures that are connected by the interface $\left(K_{33}^{\sigma}, M_{33}\right)$. For simplicity, we only show in single-level substructuring. A multi-level extension is performed for the shifted matrices through the same process which is described in [5].

By performing a block $\mathrm{LDL}^{\mathrm{T}}$ factorization of the matrix $K^{\sigma}$, i.e., $K^{\sigma}=$ $L \widehat{K}^{\sigma} L^{T}$, the shifted eigenproblem (5) is transformed to the eigenproblem

$$
\widehat{K}^{\sigma} \widehat{q}=\lambda^{\sigma} \widehat{M} \widehat{q}
$$

where $\widehat{K}^{\sigma}$ and $\widehat{M}$ are in the Craig-Bampton form [3]:

$$
\widehat{K}^{\sigma}=L^{-T} K^{\sigma} L^{-1} \quad \text { and } \quad \widehat{M}=L^{-T} M L^{-1}
$$

The next step of the AS is to extract the eigenmodes (called local modes) of the interior substructures and interface specified by the local cutoff values $\mu_{\text {min }}^{\sigma}$ and $\mu_{\max }^{\sigma}$. The subspace spanned by the column of the matrix

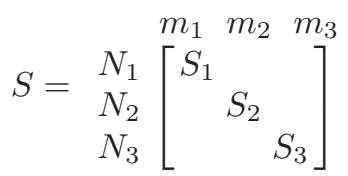

where $S_{1}, S_{2}$ and $S_{3}$ consist of extracted eigenvectors of substructures and the interface, respectively.

By projecting the eigenproblem (7) onto the subspace span $S$, then we have a reduced eigensystem of order $m=m_{1}+m_{2}+m_{3}$ :

$$
K_{m}^{\sigma} \phi=\theta^{\sigma} M_{m} \phi
$$

where $K_{m}^{\sigma}=S^{T} \widehat{K}^{\sigma} S$ and $M_{m}=S^{T} \widehat{M} S$. The eigenmodes $\phi$ are referred to as the global modes. These global modes are grouped into retained modes and truncated modes determined by (left and right) global cutoff values $\lambda_{\min }^{\sigma}$ and $\lambda_{\max }^{\sigma}$. If we write $\Phi=[\phi]=\left[\Phi_{l} \Phi_{n} \Phi_{r}\right]$, then $\Phi_{n}$ are the retained modes, $\Phi_{l}$ and $\Phi_{r}$ are the truncated modes corresponding to the eigenvalues smaller and larger than the cutoff values $\lambda_{\min }^{\sigma}$ and $\lambda_{\max }^{\sigma}$, respectively. $\Phi_{t}$ are all truncated modes, $\Phi_{t}=\left[\Phi_{l} \Phi_{r}\right]$. The subspace spanned by the columns of the matrix $L^{-1} S$ is referred to as an $A S$ subspace. 


\section{Frequency Response Analysis}

With the assumption of Rayleigh damping $D=\alpha M+\beta K$ and the introduction of the shift $\sigma$, the transfer function $H(\omega)$ can be written as

$$
H(\omega)=l^{T}\left[\gamma_{1} K^{\sigma}+\gamma_{2} M\right]^{-1} b,
$$

where $\gamma_{1}=\gamma_{1}(\omega)=1+i \omega \beta$ and $\gamma_{2}=\gamma_{2}(\omega, \sigma)=-\omega^{2}+\sigma+i \omega(\alpha+\sigma \beta)$. Projecting $H(\omega)$ onto the AS subspace, we have

$$
H_{m}(\omega)=l_{m}^{T}\left[\gamma_{1} K_{m}^{\sigma}+\gamma_{2} M_{m}\right]^{-1} b_{m}=l_{m}^{T} p_{m}(\omega),
$$

where $l_{m}=\left(L^{-1} S\right)^{T} l$ and $b_{m}=\left(L^{-1} S\right)^{T} b$, and $p_{m}(\omega)$ is the solution of the parameterized linear system of the order $m$ :

$$
G_{m}(\omega) p_{m}(\omega)=b_{m} .
$$

where $G_{m}(\omega)=\gamma_{1} K_{m}^{\sigma}+\gamma_{2} M_{m}$. It is typical that by the AS method, the order $m$ is still too large to apply for computing frequency responses. In AMLS, a so-called frequency sweep (FS) algorithm is introduced [2]. The FS algorithm retains only the low frequency modes and truncate all high frequency modes. However, for efficient Hi-FRA, it is important to be able to retain those modes corresponding to the frequency range $\left[\omega_{\min }, \omega_{\max }\right]$ of interest. To do so, let the vector $p_{m}(\omega)$ be written as $p_{m}(\omega)=p_{n}(\omega)+p_{t}(\omega)$ where $p_{n}(\omega)$ are in the subspace spanned by the retained global modes $\Phi_{n}$ and $p_{t}(\omega)$ in the subspace spanned by the truncated modes $\Phi_{l}$ and $\Phi_{r}$. Write $p_{n}(\omega)=\Phi_{n} \eta_{n}(\omega)$ for some coefficient vector $\eta_{n}(\omega)$, then the equation (12) becomes

$$
G_{m}(\omega)\left(\Phi_{n} \eta_{n}(\omega)+p_{t}(\omega)\right)=b_{m} .
$$

Pre-multiplying the equation by $\Phi_{n}^{T}$, then by the orthogonality of the global modes, the vector $p_{n}(\omega)$ is immediately given by the $n$ uncoupled equations:

$$
p_{n}(\omega)=\Phi_{n}\left(\Phi_{n}^{T} G_{m}(\omega) \Phi_{n}\right)^{-1} \Phi_{n}^{T} b_{m}=\Phi_{n}\left(\gamma_{1} \Theta_{n}^{\sigma}+\gamma_{2} I\right)^{-1} \Phi_{n}^{T} b_{m} .
$$

Subsequently, the equation (13) can be written as a parameterized linear system for $p_{t}(\omega)$ :

$$
G_{m}(\omega) p_{t}(\omega)=b_{m}-G_{m}(\omega) p_{n}(\omega) .
$$

Since it is anticipated the effect of the truncated modes for the accuracy of FRA is marginal, we employ a simple iterative refinement scheme for computing $p_{t}^{\ell-1}(\omega): p_{t}^{\ell}(\omega)=p_{t}^{\ell-1}(\omega)+\Delta p_{t}^{\ell}(\omega)$ where the correction term $\Delta p_{t}^{\ell}(\omega)$ is the solution of the refinement equation

$$
G_{m}(\omega) \Delta p_{t}^{\ell}(\omega)=r_{m}^{\ell-1}(\omega),
$$

and $r_{m}^{\ell-1}(\omega)=b_{m}-G_{m}(\omega)\left(p_{n}(\omega)+p_{t}^{\ell-1}(\omega)\right)$, the $(\ell-1)$-th residual vector.

To solve the refinement equation (16), we use a Galerkin subspace projection technique, namely, seek $\Delta p_{t}^{\ell}(\omega)$ such that

$$
\Delta p_{t}^{\ell}(\omega) \in \operatorname{span}\left\{\Phi_{t}\right\} \quad \text { and } \quad G_{m}(\omega) \Delta p_{t}^{\ell}(\omega)-r_{m}^{\ell-1}(\omega) \perp \operatorname{span}\left\{\Phi_{t}\right\} .
$$


By some algebraic manipulation, we have

$$
\begin{aligned}
\Delta p_{t}^{\ell}(\omega) & =\Phi_{t}\left(\gamma_{1} \Theta_{t}^{\sigma}+\gamma_{2} I\right)^{-1} \Phi_{t}^{T} r_{m}^{\ell-1}(\omega) \\
& =\left[\left(\gamma_{1} K_{m}^{\sigma}+\gamma_{2} M_{m}\right)^{-1}-\Phi_{n}\left(\gamma_{1} \Theta_{n}^{\sigma}+\gamma_{2} I\right)^{-1} \Phi_{n}^{T}\right] r_{m}^{\ell-1}(\omega) .
\end{aligned}
$$

For computational efficiency, noting that $K_{m}^{\sigma}$ is diagonal, we simply use the following approximation for computing the correction term $\Delta p_{t}^{\ell}(\omega)$ :

$$
\Delta p_{t}^{\ell}(\omega) \approx \Phi_{t}\left(\gamma_{1} \Theta_{t}^{\sigma}\right)^{-1} \Phi_{t}^{T} r_{m}^{\ell-1}(\omega)
$$

Subsequently, we derive the following iterative refinement iteration for computing the vector $p_{t}(\omega)$ :

$$
p_{t}^{\ell}(\omega)=p_{t}^{\ell-1}(\omega)+\frac{1}{\gamma_{1}}\left[\left(K_{m}^{\sigma}\right)^{-1}-\Phi_{n}\left(\Theta_{n}^{\sigma}\right)^{-1} \Phi_{n}^{T}\right] r_{m}^{\ell-1}(\omega)
$$

for $\ell=1,2, \ldots$, with the initial guess $p_{t}^{0}(\omega)$. A practical stopping criterion is to test the relative residual error $\left\|\Delta p_{t}^{\ell}(\omega)\right\|_{2} /\left\|\left(\gamma_{1} K_{m}^{\sigma}\right)^{-1} b_{m}\right\|_{2} \leq \epsilon$ for a given tolerance $\epsilon$. The convergent solution is denoted as $p_{t}^{*}(\omega)$.

Assume that it is required to calculate the $n_{f}$ frequency points: $\omega_{\min } \leq \omega_{1}<$ $\omega_{2}<\cdots<\omega_{n_{f}} \leq \omega_{\max }$. Then we can determine the initial guess $p_{t}^{0}\left(\omega_{k}\right)$ at the frequency $\omega_{k}$ by a linear extrapolation for $k=3,4, \ldots, n_{f}$ with $p_{t}^{0}\left(\omega_{1}\right)=0$ and $p_{t}^{0}\left(\omega_{2}\right)=p_{t}^{*}\left(\omega_{1}\right)$. Then all initial guess $p_{t}^{0}\left(\omega_{k}\right) \in \operatorname{span}\left\{\Phi_{t}\right\} \cdot p_{t}^{\ell}(\omega)$ by the iteration (18) is guaranteed to be orthogonal to the vector $p_{n}(\omega)$.

Now we turn to investigate the relationship between the frequency range $\left[\omega_{\min }, \omega_{\max }\right]$ of interest and the interval $\left[\lambda_{\min }^{\sigma}, \lambda_{\max }^{\sigma}\right]$ for the global modes to be retained to guarantee the convergence of the iteration (18). By the equation (17), we have

$$
\left\|\Delta p_{t}^{\ell}(\omega)\right\|_{2} \approx\left\|\Phi_{t}\left(\gamma_{1} \Theta_{t}^{\sigma}\right)^{-1} \Phi_{t}^{T} r_{m}^{\ell-1}(\omega)\right\|_{2} \leq\left\|\Phi_{t}\left(\gamma_{1} \Theta_{t}^{\sigma}\right)^{-1}\right\|_{2}\left\|\Phi_{t}^{T} r_{m}^{\ell-1}(\omega)\right\|_{2}
$$

The term $\Phi_{t}^{T} r_{m}(\omega)$ is referred to as a truncated modal residual. By some algebraic manipulation, we see that two consecutive truncated modal residuals satisfy the relation

$$
\Phi_{t}^{T} r_{m}^{\ell}(\omega)=-\frac{\gamma_{2}}{\gamma_{1}}\left[\begin{array}{ll}
\Theta_{l}^{\sigma} & \\
& \Theta_{r}^{\sigma}
\end{array}\right]^{-1} \Phi_{t}^{T} r_{m}^{\ell-1}(\omega)
$$

Therefore, if we introduce a positive constant $\xi$, referred to as contraction ratio, such that

$$
\left|\frac{\phi_{k}^{T} r_{m}^{\ell}(\omega)}{\phi_{k}^{T} r_{m}^{\ell-1}(\omega)}\right|=\frac{d(\omega, \sigma)}{\left|\theta_{k}^{\sigma}\right|} \leq \frac{d_{\max }}{\left|\theta_{k}^{\sigma}\right|} \leq \xi<1
$$

where $\phi_{k} \in \Phi_{t}, d(\omega, \sigma)=\left|-\gamma_{2} / \gamma_{1}\right|$, and $d_{\max }=\max \left\{d\left(\omega_{k}, \sigma\right), 1 \leq k \leq n_{f}\right\}$. Then the components of the truncated modal residual are contracted, i.e., the norm of the correction term $\Delta p_{t}(\omega)$ decreases and the iteration (18) converges. 
By (19), it derives that the global modes outside the interval $\left[-d_{\max } / \xi, d_{\max } / \xi\right]$ can be "cut off", i.e., the global cutoff values are determined by

$$
\lambda_{\min }^{\sigma}=-d_{\max } / \xi \quad \text { and } \quad \lambda_{\max }^{\sigma}=d_{\max } / \xi
$$

When there is no shift, i.e., $\sigma=0$, the low global cutoff value is less than zero. It means that all low frequency modes smaller than $\lambda_{\max }^{\sigma}$ are retained.

\section{ASFRA Algorithm}

By combining the AS method for extracting eigenpairs and the frequency sweep iteration, we derive an algorithm for computing the frequency responses $H\left(\omega_{k}\right)$. The algorithm is referred to as the ASFRA algorithm. In this section, we briefly discuss the choice of parameters in ASFRA. Detail will be presented in a full paper elsewhere.

It is necessary that the shift $\sigma \in\left[\omega_{\min }^{2}, \omega_{\max }^{2}\right]$. In order to minimize the range of the global modes to be retained/extracted, the center of the frequency range is used, $\sigma=\frac{1}{2}\left(\omega_{\max }^{2}+\omega_{\min }^{2}\right)$.

By (20), the global cutoff values $\lambda_{\min }^{\sigma}$ and $\lambda_{\max }^{\sigma}$ are essentially determined the contraction ratio $\xi$. To improve the convergence of the FS iteration (18), the contraction ratio $\xi$ should be small. However, it makes the number of retained global modes large. From our numerical experiments, we found that a good choice is $\xi=0.5$.

How to retain the local modes for a desired number and accuracy of global modes has been an important issue in the study of the AS algorithm [10. To achieve a desired level of accuracy of the global modes, a large number of local modes are required. the local cutoff values $\mu_{\min }^{\sigma}$ and $\mu_{\max }^{\sigma}$ are typically chosen proportionally to the global cutoff values $\lambda_{\min }^{\sigma}$ and $\lambda_{\max }^{\sigma}$, namely $\mu_{\min }^{\sigma}=c_{l} \lambda_{\min }^{\sigma}$ and $\mu_{\max }^{\sigma}=c_{u} \lambda_{\max }^{\sigma}$, where $c_{l}$ and $c_{u}$ are relaxation factors. As $c_{l}$ and $c_{u}$ increase, the accuracy of the global modes is typically improved. We use $c_{l}=c_{u}=10$ as default by referring to the previous research in [1].

We implemented ASFRA based on the ASEIG [5. The multilevel partition is done by METIS [6]. The global modes and the local modes of the substructure blocks are computed by ARPACK [7] with SuperLU [4] and the local modes of the interface are solved by LAPACK.

We will compare the performance of ASFRA with three other methods in the next section. Let us briefly review these methods. The first method is the so-called direct method. It computes the frequency responses $H\left(\omega_{k}\right)$ by solving the underlying linear system (2) by a direct sparse method. Specifically, we use the SuperLU method. The second method is to use the shift-and-invert Lanczos (SIL) method from ARPACK to extract $n$ eigenmodes and then approximate $H\left(\omega_{k}\right)$ by $H_{n}\left(\omega_{k}\right)$ as defined in (4). The shift is $\sigma=0$ and the eigenmodes are determined by upper cutoff value $\lambda_{\max }$ and the residual flexibility vectors are supplemented [9]. The upper cutoff value $\lambda_{\max }$ is determined by $\lambda_{\max }=$ $\left(\chi \omega_{\max }\right)^{2}$, where $\chi$ is a multiplication factor. Typically, $\chi=2$ or 3 , when there are no residual flexibility vectors. Otherwise, $\chi$ can be smaller, say 1.11. The 


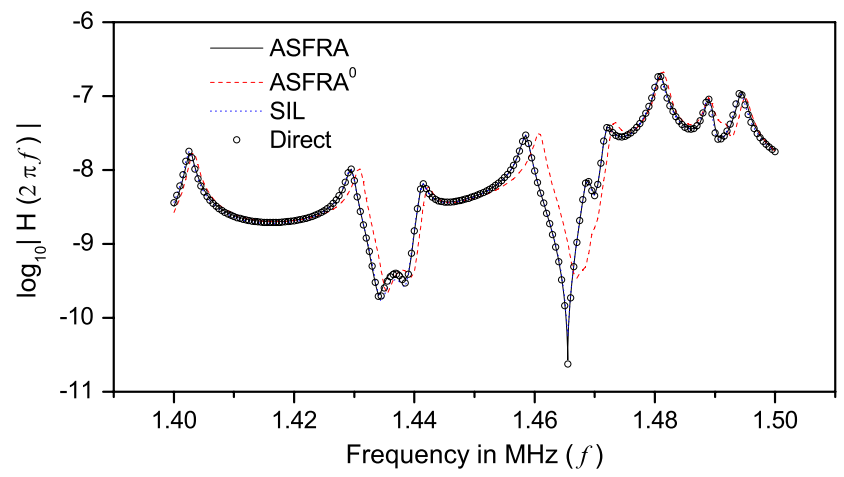

Fig. 1. The frequency responses of a butterfly gyro

third method is a special case of ASFRA with the zero shift $\sigma=0$, and the lower cutoff value $\lambda_{\min }^{\sigma}=0$. The frequency response $H\left(\omega_{k}\right)$ is approximated by $H_{m}\left(\omega_{k}\right)$ in eq. (11). It is denoted as $\mathrm{ASFRA}^{0}$. ASFRA ${ }^{0}$ is essentially the AMLS with frequency sweep iteration as presented in [2].

\section{Numerical Experiment}

MEMS resonators under an electrostatic actuation are utilized in various MEMS devices such as angular rate sensors and bandpass filters. Specifically, we consider a FE model with solid elements of a butterfly gyro which is an angular rate sensor using the MEMS resonator [8]. The order of the system $K$ and $M$ matrices is $N=17631$. The Rayleigh damping parameters are set by $\alpha=0.0, \beta=$ $10^{-10}$. Frequency responses changes rapidly near the resonances in the range $\left[f_{\min }, f_{\max }\right]=\left[\omega_{\min } /(2 \pi), \omega_{\max } /(2 \pi)\right]=[1.4,1.5] \mathrm{MHz}$. Numerical experiment is conducted on an Intel Itanium 2 Server with Linux OS.

The substructuring level of the AS is 3. The tolerance of the frequency sweep iteration is $\epsilon=10^{-5}$. By Figure 1. ASFRA shows better accuracy than ASFRA ${ }^{0}$ with the given parameters. The detailed results are listed in Table 1 . All methods calculate the responses at $n_{f}=201$ frequencies $\omega_{k}$ in an equal space on the interval. ASFRA is 2.3 times faster than $\mathrm{ASFRA}^{0}$. SIL is more expensive than ASFRA because it needs to compute eigenmodes of the full-size eigensystem. Finally, we note that the performance of ASFRA and ASFRA ${ }^{0}$ does not change significantly when the parameters $\xi$ and $c_{l}, c_{u}$ are changed slight from the present choice.

Table 1. The dimension of AS subspace, numbers of retained modes, total FS iteration, and the elapsed time

\begin{tabular}{lcccc}
\hline & ASFRA & ASFRA & SIL & Direct \\
\hline$m$ (AS subspace dim.) & 213 & 651 & - & - \\
$n$ (retained modes) & 20 & 175 & 156 & - \\
Total FS iteration & 238 & 51 & - & - \\
Elapsed Time(sec.) & 26.77 & 62.94 & 80.42 & 754.6 \\
\hline
\end{tabular}




\section{Conclusion}

In this paper, we presented an algebraic substructuring based frequency response analysis (ASFRA) algorithm to calculate the frequency response of a large dynamic system between two specified frequency $\omega_{\min }$ and $\omega_{\max }$. ASFRA can be efficiently applied to Hi-FRA, as demonstrated by a microelectomechanical sensor operated at $1 \mathrm{MHz}-2 \mathrm{MHz}$. Future work includes the optimal choice of parameters and parallelization techniques.

Acknowledgments. JHK was supported by Korea Research Foundation Grant KRF-2005-214-D00015. ZB was supported in part by the NSF grant DMS0611548. Most of this work was done while JHK visiting the University of California, Davis.

\section{References}

1. Bennighof, J. K., Kim, C. K. (eds.): An addaptive multi-level substururing method for efficient modeling of complex strucutures. Proceedings of the AIAA 33rd SDM Conference, Dallas, Texas, (1992) 1631-1639

2. Bennighof, J. K., Kaplan, M. F. (eds.): Frequency sweep analysis using multi-level substructuring, global modes and iteration. Proceedings of 39th AIAA/ASME/ASCE/AHS Structures, Structural Dynamics and Materials Conference. (1998)

3. Craig, Jr. R. R., Bampton, M.C.C. (eds.): Coupling of substructures for dynamic analysis. AIAA Journal, Vol. 6(7). (1968) 1313-1319

4. Demmel, J.W., Eisenstat, S. C., Gilbert, J. R. , Li, X. S., Liu, J. W. H. (eds.): A supernodal approach to sparse partial pivoting. SIAM J. Matrix Anal. Appl., Vol. 20(3). (1999) 720-755

5. Gao, W., Li, X.S., Yang, C., Bai, Z. (eds.): An implementation and evaluation of the AMLS method for sparse eigenvalue problems. Technical Report LBNL-57438, Lawrence Berkeley National Laboratory. (2006)

6. Karypis, G.: METIS. Department of Computer Science and Engineering at the University of Minnesota, http://wwwusers.cs.umn.edu/ karypis/metis/metis/index.html. (2006)

7. Lehoucq, R., Sorensen, D. C., Yang, C.: ARPACK User's Guide: Solution of LargeScale Eignevalue Problems with Implicitly Restarted Arnoldi Methods. SIAM. Philadelphia (1998)

8. Lienemann, J., Billger, D., Rudnyi, E. B., Greiner, A., Korvink, J. G.: MEMS compact modeling meets model order reduction: Examples of the application of arnoldi methods to microsystem devices. The Technical Proceedings of the 2004 Nanotechnology Conference and Trade Show, Nanotech 04. (2004)

9. Thomas, B., Gu, R. J.: Structural-acoustic mode synthesis for vehicle interior using finite-boundary elements with residual flexibility. Int. J. of Vehicle Design, Vol. 23. (2000) 191-202

10. Yang, C., Gao, W., Bai, Z., Li, X., Lee, L., Husbands, P., Ng, E.: An algebraic substructuring method for large-scale eigenvalue calculations. SIAM J. Sci. Comput., Vol. 27(3). (2005) 873-892 